\title{
SY27-3 Symposium
}

\section{KANPHOS (Kinase-Associated Neural PHOspho-Signaling), a comprehensive database for specific substrates of kinases}

\author{
Kozo Kaibuchi ${ }^{1}$, Mutsuki Amano ${ }^{1}$, Takaki Nishioka ${ }^{1}$, Junichiro Yoshimoto ${ }^{2}$

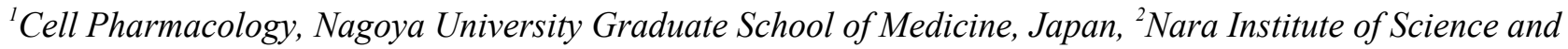 \\ Technology, Japan
}

More than 500 protein kinases are encoded in the human genome. Although several kinases have been well-characterized and demonstrated to be physiologically important, the functions of a number of kinases remain to be elucidated. To identify the specific substrates for the specific kinases, we have recently developed an in vitro approach termed the kinaseinteracting substrate screening (KISS) method (Amano et al. J Cell Biol 2015) and an in vivo approach termed kinaseoriented substrate screening (KIOSS) method (Nagai et al. Neuron 2016; Nagai et al. Trend Pharmacol Sci 2016). By using these methods, we identified the PKA substrates downstream of dopamine from mouse striatum, and found more than one hundred candidate substrates of PKA, including Rap1 GEF (Rasgrp2). We also found that PKA phosphorylation of Rasgrp2 activated its guanine nucleotide exchange activity on Rap1. Cocaine exposure activated Rap1 in nucleus accumbense in mice through dopamine. The expression of constitutively active PKA or Rap1 in accumbal D1R-expressing medium spiny neurons (D1R-MSNs) enhanced neuronal firing rates and behavioral responses to cocaine exposure through MAPK. These findings demonstrate a novel DA-PKA-Rap1-MAPK intracellular signaling mechanism in D1RMSNs that increases neuronal excitability to enhance reward-related behaviors. We also constructed an on-line database system, named KANPHOS (Kinase-Associated Neural PHOspho-Signaling), that provides the phosphorylation signals identified by our methods as well as those previously reported in the literature. We here discuss the phospho-proteomic approach and the database. 